\title{
Initial Experience with Neuroform EZ in the Treatment of Wide-neck Cerebral Aneurysms
}

\author{
Erwin Zeta Mangubat, MD, MPH, Andrew Kelly Johnson, MD, \\ Kiffon M. Keigher, APN, Demetrius Klee Lopes, MD
}

Purpose: Stent-assisted coiling allows embolization and parent vessel reconstruction of wide-necked intracranial aneurysms. The Neuroform EZ (Boston Scientific, Fremont, CA, U.S.A.) stent delivery system offers deployment of a Neuroform stent with fewer steps and improved operator control. Initial experience, technical considerations, and treatment outcomes using the Neuroform EZ stent delivery system in combination with coil embolization are reported.

Materials and Methods: Seventeen consecutive patients harboring 21 wide-necked saccular cerebral aneurysms were treated with stent reconstruction. Twenty aneurysms were unruptured; one was treated within 24 hours of diagnosis of rupture. Twenty aneurysms were located in the anterior circulation; one was in the posterior circulation. Immediate and six-month post-treatment angiography and clinical assessment were performed.

Results: In all cases, the stents were delivered and positioned without difficulty in deployment. Technical complications occurred in 4 patients, but none were directly related to the stent delivery system. On immediate post-treatment angiography, 5 of 21 aneurysms showed complete occlusion, 5 of 21 showed residual neck, and 11 of 21 showed residual contrast filling of the aneurysm sac. At six month follow-up, all 17 patients were clinically stable. Angiography of 18 of the aneurysms showed total occlusion in 12, residual neck in 3, and residual aneurysm filling in 3. Retreatment was performed in the three with residual aneurysm.

Conclusion: The Neuroform EZ stent system offers improved anchoring and support in stent delivery, which is particularly useful when multiple stents are overlapped to further protect the parent vessel and increase flow diversion away from the aneurysm sac. The only significant problem encountered was coil prolapse, which could be treated with a second stent when necessary. The ease of deployment improves upon the already clinically successful Neuroform design.

Key Words : Aneurysm; Coil embolization; Neuroform EZ; Stent-assisted coiling

All authors: Department of Neurosurgery, Rush University Medical Center, Chicago, Illinois, U.S.A.

Received January 24, 2012; accepted after revision February 20, 2012.

Correspondence to: Demetrius K. Lopes, MD, Department of Neurological Surgery, Rush University Medical Center, 1725 West Harrison St. Suite 970, Chicago, Illinois 60612, U.S.A.

E-mail: brainaneurysm@mac.com

This is an Open Access article distributed under the terms of the Creative Commons Attribution Non-Commercial License (http://creativecommons.org/licenses/by-nc/3.0) which permits unrestricted non-commercial use, distribution, and reproduction in any medium, provided the original work is properly cited. 
Stent-assisted coiling for treatment of wide-necked intracranial aneurysms has grown in popularity. Selfexpanding stents provide mechanical, hemodynamic, and biological benefits by preventing coil protrusion into the parent artery, reducing intra-aneurysmal flow, and promoting vessel wall healing $[1,2]$. The introduction of the first flexible intracranial Neuroform stent (Boston Scientific, Fremont, CA, U.S.A.) and the subsequent preliminary results have shown this device to be an effective tool in treating pathological entities that were previously not considered amenable to endovascular therapy [3-9]. The Neuroform 3 Stent Delivery System (previous version) is a preloaded stent within a microcatheter that must be advanced en bloc or transferred to a pre-positioned alternate microcatheter. En bloc advancement can be difficult in tortuous anatomy, and stent transfer is time consuming, wasteful, and complicated for a single operator. In this article, we report our initial clinical experience, technical considerations, and treatment outcomes in 17 consecutive patients harboring a total of 21 widenecked aneurysms using the Neuroform EZ Stent Delivery System, which incorporates a simplified, single-operator delivery system intended to facilitate stent preparation, delivery, and positioning.

\section{PATIENTS AND METHODS}

\section{Demographic data and inclusion criteria}

Seventeen consecutive adult patients with 21 widenecked intracranial saccular aneurysms were included in the study. Prior to treatment, the neurovascular team considered the options of observation, open surgical and endovascular repair in each case. Angiographic studies obtained in all aneurysms were reviewed by the attending endovascular neurosurgeon (D.K.L). All saccular bifurcation aneurysms included in the study had a wide neck, defined as a dome-to-neck ratio of less than 2 or a neck that was $4 \mathrm{~mm}$ or wider as measured on angiography $[10,11]$. A signed informed consent was obtained for each patient. Demographic data obtained and recorded for each patient included clinical history, aneurysm location and dimensions, technical difficulties, and complications. Immediate post-procedure angiography was performed to determine degree of aneurysm occlusion and parent vessel patency. A frequently updated database with the aforementioned data was retrospectively reviewed. This study was conducted in accordance to and with approval by the Rush University Medical Center Institutional Review Board.

\section{Patient population}

Between June, 2010 and September, 2010, we deployed 30 Neuroform EZ stents in 17 patients (12 women) for treatment of 21 wide-necked cerebral aneurysms. The mean age at the time of treatment was 56.3 years (range 37-79 years). Stents were deployed in an overlapping manner in 7 patients, and a y-stent configuration was used to reconstruct the parent vessels of 2 aneurysms $[12,13]$. There were 3 aneurysms located at the cavernous carotid segment, 3 at the origin of the ophthalmic artery, 4 at the superior hypophyseal artery, 4 at the posterior communicating artery, 1 along the posterior carotid wall, 1 on the A1 segment of anterior cerebral artery, 2 at the anterior communicating artery, 2 at the middle cerebral artery bifurcation, and 1 at the basilar bifurcation. The average maximum diameter of the 21 aneurysms was $8.3 \mathrm{~mm}$ (range 2.5$24.0 \mathrm{~mm}$ ) with average neck $5.7 \mathrm{~mm}$ (range 2.0-17.9 $\mathrm{mm}$ ). Three aneurysms initially treated via coiling only were found to have recurrent filling on 6-month followup; these were treated via stent-assisted coiling with the Neuroform EZ stent and included in the study. In unruptured and previously treated aneurysms, the Neuroform EZ stent was placed across the neck of the lesion prior to coil embolization. In the case of the one acutely ruptured aneurysm, a Neuroform EZ stent was deployed after coil embolization as post-coiling angiography demonstrated parent artery compromise.

\section{Stent delivery method}

Patients treated electively receive anti-platelet pharmacotherapy (325 mg aspirin and $75 \mathrm{mg}$ clopidogrel daily) starting 5 days before the procedure. The one patient who presented with subarachnoid hemorrhage was loaded with anti-platelet medication following the procedure. Intra-operative heparinization to an activated clotting time of 250 seconds is maintained throughout the procedure. Dual anti-platelet therapy continues for three months after the procedure and for six months when more than one stent is placed.

After diagnostic angiography is performed, working views are obtained for stent delivery. The appropriate Neuroform EZ stent length is selected (lengths available were 10, 15, 20 and $30 \mathrm{~mm}$ ) to allow the ends of the device to extend at least $5 \mathrm{~mm}$ beyond the neck of the aneurysm on each side. All stents deployed were $4.5 \mathrm{~mm}$ in width to maximize vessel apposition and increase metal to artery ratio.

A guide catheter is positioned in the cervical segment of the target vessel. A microcatheter (Renegade Hiflow (Stryker) or Marksman (Covidien)) is navigated 
as distally as possible beyond the aneurysm neck. The Neuroform EZ stent loading sheath is introduced into a rotating hemostatic valve connected to the microcatheter and continuous heparinized saline flush. Back flow of saline from the valve preps the system through the loading sheath. The loading sheath is inserted to the hub of the microcatheter and the rotating hemostatic valve is tightened around the sheath. The stent is introduced into the microcatheter under direct visualization through the transparent tip of the loading sheath. The stent and delivery system are then advanced beyond the aneurysm neck within the microcatheter; the microcatheter is withdrawn while keeping forward pressure on the stent delivery system, unsheathing the stent across the neck of the aneurysm. The catheter can be manipulated forward and backward to control speed of deployment and expansion of each open cell unit.

Visualization of the four platinum marker bands at each end of the stent confirms proximal and distal placement within the parent vessel. After stent deployment, a Synchro 14 (Stryker) microguidewire and SL 10 microcatheter are advanced through the interstices of the stent into the aneurysm to allow coil embolization.

\section{Anatomic and clinical outcome assessment}

Patients were evaluated by angiography at the end of the procedure to document aneurysm obliteration. A qualitative assessment of aneurysm occlusion was performed using a 3-point Raymond scale [14]: 1) complete aneurysm occlusion without contrast filling; 2 ) residual neck; and 3) residual aneurysm sac. In all cases, aneurysm occlusion was interpreted on the basis of the images obtained immediately after placement of the last coil. The senior author evaluated the clinical outcome at discharge and follow-up with modified Rankin score (mRS) [15]. Six-month follow-up angiography was performed on 18 of the 21 treated aneurysms. Two patients with a total of 3 aneurysms are clinically stable but are still awaiting angiographic follow-up.

\section{RESULTS}

Precise delivery of the stent was successfully performed in all cases. Navigation of the stent delivery system was accomplished without difficulty through tortuous internal carotid and vertebral arteries. There were no technical complications with stent delivery. In cases where multiple stents were positioned in an overlapping fashion, the microcatheter could be readvanced over the stent-deployment wire through the deployed stent. After deployment, no stents required retrieval or repositioning. No artery branch occlusion due to intra-stent thrombus was demonstrated angiographically, and no thromboembolic complications, arterial dissections, or arterial ruptures occurred during stent placement.

All of the aneurysms both ruptured and unruptured underwent coil embolization in the same setting as stent placement. Navigation of the microcatheter through the interstices of the stent was easily accomplished in all cases. Microcatheter manipulation during coil embolization of the aneurysm did not cause stents to move from the site of original placement.

On immediate post-embolization angiography, 5 aneurysms showed complete occlusion, 5 showed contrast filling the neck of the aneurysm, and 12 showed contrast filling the sac of the aneurysm (Table 1).

\section{Technical Difficulties}

Technical complications occurred in 4 patients, including prolapsed coils, vasospasm, and parent vessel compromise, all of which had no clinical significance.

In case 5, a Neuroform EZ stent was placed across a wide-necked superior hypophyseal artery aneurysm followed by coiling. Unfortunately, the first coil stretched and prolapsed into the parent vessel. An Enterprise stent (Codman, Miami Lakes, FL, U.S.A.) was deployed, overlapping the Neuroform EZ stent to keep the prolapsed coil segment out of the vessel

Table 1. Angiographic Results

\begin{tabular}{|c|c|c|c|c|c|c|c|}
\hline & \multicolumn{3}{|c|}{ Initial Raymond Class } & \multicolumn{3}{|c|}{ 6-month follow-up Raymond Class* } & \multirow{2}{*}{ Retreat } \\
\hline & I & II & III & I & II & III & \\
\hline Total & 5 & 5 & 11 & 12 & 3 & 3 & 3 \\
\hline$<10 \mathrm{~mm}$ & 3 & 4 & 8 & 11 & 2 & 0 & 0 \\
\hline$>10 \mathrm{~mm}$ & 2 & 1 & 3 & 1 & 1 & 3 & 3 \\
\hline
\end{tabular}

* Follow-up angiography unavailable for three patients who are known to be clinically stable. 
lumen. In case 8, after deployment of a Neuroform EZ stent across the neck of a carotid ophthalmic artery aneurysm and carotid cavernous aneurysm, coil packing of the two separate aneurysms through the stent initially resulted in partial prolapse of coil mass into the parent vessel. A second Neuroform EZ stent was placed successfully. Post-procedure angiography demonstrated no parent vessel compromise in these two cases, nor were there any clinical sequelae.

In case 13, after coiling a basilar tip aneurysm, postprocedure angiography demonstrated prolapsed coils, occluding the small left posterior cerebral artery. A Neuroform EZ stent was placed from the right posterior cerebral artery to basilar artery. Prior to closure, a left common carotid artery angiogram demonstrated a fetal origin of the left posterior cerebral artery. The patient remained neurologically intact.

In case 14 , while treating a wide-necked anterior communicating artery aneurysm, there was iatrogenic vasospasm resulting in mild changes in somatosensory evoked potentials. Potentials returned to baseline after use of intra-arterial nicardipine and blood pressure augmentation. The patient had no new neurologic deficits after the procedure.

\section{Clinical Outcome}

Selective embolization was successfully performed and resulted in an excellent clinical outcome in all patients. One patient had a groin hematoma without evidence of pseudoaneurysm or arteriovenous fistula on ultrasound and without clinical sequelae. One patient had elevated troponins post-procedure related to demand ischemia without clinical consequence. There were no treatment-related deaths or clinically evident neurological complications. The one patient who presented in the acute stage of subarachnoid hemorrhage (ruptured basilar tip aneurysm) did well post-operatively and was discharged home on postoperative day 11. All seventeen patients were discharged home with a good clinical status (mRS $0, n$ $=11 ; \operatorname{mRS} 1, \mathrm{n}=6)$. All patients were clinically evaluated approximately six months after aneurysm treatment, and none had evidence of treatment-related complications.

Follow-up angiography (Table 1) showed total occlusion in 12 , residual neck in 3 , and residual aneurysm filling in 3 . Retreatment was performed for the three aneurysms with residual filling. Two of these had further coiling for coil compaction without complication; follow-up angiography of these retreatments is pending. The third patient also had further coiling for compaction inside a particularly challenging partially thrombosed large wide-neck aneurysm; there was continued residual filling on his subsequent follow-up. A third procedure involving non-Neuroform stent was performed on this patient which was complicated by a late intracerebral hemorrhage despite Raymond class 1 occlusion on post-intracranial hemorrhage angiography. There was no significant stenosis within the stent constructs of the eighteen aneurysms with follow-up. We continue to follow all patients in this series, and to our knowledge, no others have had aneurysm-related clinical problems.

\section{DISCUSSION}

The development of self-expanding intracranial stents has increased the options for treatment of wide-necked aneurysms. The original Neuroform device was introduced as the first flexible stent made of a nickeltitanium alloy (nitinol) designed for treatment of intracranial wide-necked and fusiform aneurysms. This device has been evaluated in a number of preliminary studies [3, 6, 10, 16-19], demonstrating its efficacy, safety, and satisfactory immediate anatomical results. The largest series of Neuroform stents in the treatment of aneurysms was recently published with excellent results [20], and our experience with over 300 Neuroform stents mirrors this success. The present Neuroform EZ series attests to the ease of using the Neuroform EZ stent system, as well as the good anatomical and clinical outcomes associated with other Neuroform stents.

Compared to the Neuroform 3 delivery system, which required en bloc advancement of catheter and loaded stent, the Neuroform EZ allows the operator to select their desired micro-catheter without stent transfer. The operator may obtain access with their choice of microwire and microcatheter and avoid the difficulty of en bloc advancement through tortuous anatomy. The bumpers allow the stent to be moved backward and forward prior to unsheathing to achieve optimal positioning and microcatheter stability prior to deployment. The stent-deployment wire has a tip that extends beyond the microcatheter, and while this nonnavigable tip could potentially cause difficulty in stent advancement, it was not a problem in our experience due to adequate distal microcatheter access. Placement of overlapping stents requires fewer steps because the microcatheter and wire are left in place for loading subsequent stents, providing a key advantage over the Neuroform 3 design. 
The Neuroform EZ design is an excellent option for most intracranial aneurysms, especially those with tight curves in the parent vessel or located at bifurcation. Though rare, limitations of microcatheter access can favor another stent. The Neuroform 3 design may have an advantage when insufficient distal microcatheter access is obtained. The old design does not have a leading wire on the stent deployment wire, so it can be pushed out of the microcatheter with less distal access. We sometimes use other stents like the Enterprise when only smaller microcatheters can access the aneurysm.

The duration of anti-platelet therapy is not standardized, but our strategy maintains dual anti-platelet therapy for three months when a single stent is placed. Complete endothelialization over a Neuroform stent has been demonstrated in a similar time frame [2]. When using overlapping stents, we suspect that the thromboembolic risk and time to endothelialization are both increased; therefore, a six-month or longer dual antiplatelet strategy is utilized. This may also be related to the type of aneurysm being treated. Arteries with segmental wall defects usually will require aneurysms to be treated with overlapping stents to reconstruct anatomy and avoid coil prolapse. It is unclear to date if these stents will become completely "endothelialized". Aneurysms resulted from focal arterial wall weakness usually require one stent reconstruction and are more likely to become fully "endothelialized". Antiplatelet therapy in acute subarachnoid hemorrhage complicates management. Unless absolutely necessary, we avoid or delay placing stents when the patient is likely to have other procedures including ventriculostomy. Antiplatelet strategy for stenting in the setting of acute hemorrhage have been previously reported [21].

Clinical and angiographic follow-up revealed excellent results in this preliminary study. We were fortunate to have no significant complications from Neuroform EZ procedures to this point. Furthermore, the aneurysm population showed a trend toward progressive thrombosis after treatment. While three large aneurysms required retreatment for coil compaction, we were satisfied with angiographic results overall. A larger series and longer follow-up are needed to elucidate the long-term efficacy and prognosis for aneurysms treated with the Neuroform EZ stent delivery system, but the initial results are promising. Stent deployment was easier using the newer system, and we anticipate clinical outcomes to at least match those of prior Neuroform generations. We validate with this report the safety of the transfer technique for intracranial stenting.
In conclusion, the Neuroform EZ stent delivery system is feasible, safe, and effective in treating widenecked intracranial aneurysms. In comparison to previous generations, the Neuroform EZ stent system offers improved stability and support in stent delivery to the aneurysm site, which is especially useful for overlapping multiple stents. The one recurrent technical problem of coil prolapse was managed with deployment of another stent when necessary. Though limited in scale, this study is encouraging because it demonstrates that devices like the Neuroform EZ stent delivery system may continue to improve our ability to treat wide-necked aneurysms with lower morbidity, mortality, and re-treatment rates.

Conflict of Interest: Demetrius Lopes discloses that he is a paid consultant to and receives grant/research funding from Stryker Neurovascular. The other authors have no disclosure.

\section{References}

1. Wanke I, Forsting M. Stents for intracranial wide-necked aneurysms: more than mechanical protection. Neuroradiology 2008;50:991-998

2. Lopes D, Sani S. Histological postmortem study of an internal carotid artery aneurysm treated with the Neuroform stent. Neurosurgery 2005;56:E416

3. Benitez RP, Silva MT, Klem J, Veznedaroglu E, Rosenwasser RH. Endovascular occlusion of wide-necked aneurysms with a new intracranial microstent (Neuroform) and detachable coils. Neurosurgery 2004;54:1359-1367

4. Broadbent LP, Moran CJ, Cross DT,3rd, Derdeyn CP. Management of neuroform stent dislodgement and misplacement. AJNR Am J Neuroradiol 2003;24:1819-1822

5. Fiorella D, Albuquerque FC, Deshmukh VR, Woo HH, Rasmussen PA, Masaryk TJ, et al. Endovascular reconstruction with the Neuroform stent as monotherapy for the treatment of uncoilable intradural pseudoaneurysms. Neurosurgery 2006;59: 291-300

6. Fiorella D, Albuquerque FC, Han P, McDougall CG. Preliminary experience using the Neuroform stent for the treatment of cerebral aneurysms. Neurosurgery 2004;54:6-16

7. Howington JU, Hanel RA, Harrigan MR, Levy EI, Guterman LR, Hopkins LN. The Neuroform stent, the first microcatheterdelivered stent for use in the intracranial circulation. Neurosurgery 2004:54:2-5

8. Wanke I, Doerfler A, Schoch B, Stolke D, Forsting M. Treatment of wide-necked intracranial aneurysms with a self-expanding stent system: initial clinical experience. AJNR Am J Neuroradiol 2003;24:1192-1199

9. Yun JH, Cho CS. Experiences of neuroform stent applications for ruptured anterior communicating artery aneurysms with small parent vessel. J Korean Neurosurg Soc 2010;48:53-58

10. Alfke K, Straube T, Dorner L, Mehdorn HM, Jansen O. Treatment of intracranial broad-neck aneurysms with a new self-expanding 


\section{Neuroform EZ for wide-neck Aneurysms}

stent and coil embolization. AJNR Am J Neuroradiol 2004;25:584-591

11. Lylyk P, Cohen JE, Ceratto R, Ferrario A, Miranda C. Endovascular reconstruction of intracranial arteries by stent placement and combined techniques. J Neurosurg 2002;97:13061313

12. Spiotta AM, Gupta R, Fiorella D, Gonugunta V, Lobo B, Rasmussen PA, et al. Mid-term results of endovascular coiling of wide-necked aneurysms using double stents in "Y-configuration". Neurosurgery 2011;69:421-429

13. Sani S, Lopes DK. Treatment of a middle cerebral artery bifurcation aneurysm using a double neuroform stent "Y" configuration and coil embolization: technical case report. Neurosurgery 2005;57(1 Suppl):E209

14. Raymond J, Roy D. Safety and efficacy of endovascular treatment of acutely ruptured aneurysms. Neurosurgery 1997;41:1235-1245

15. Rankin J. Cerebral vascular accidents in patients over the age of 60. II. Prognosis. Scott Med J 1957;2:200-215

16. Biondi A, Janardhan V, Katz JM, Salvaggio K, Riina HA, Gobin YP. Neuroform stent-assisted coil embolization of wide-neck intracranial aneurysms: strategies in stent deployment and midterm follow-up. Neurosurgery 2007;61:460-468

17. Gurian JH, Martin NA, King WA, Duckwiler GR, Guglielmi G, Vinuela F. Neurosurgical management of cerebral aneurysms following unsuccessful or incomplete endovascular embolization. J Neurosurg 1995;83:843-853

18. Henkes H, Bose A, Felber S, Miloslavski E, Berg-Dammer E, Kuhne D. Endovascular coil occlusion of intracranial aneurysms assisted by a novel self-expandable nitinol microstent (Neuroform). Interv Neuroradiol 2002;8:107-119

19. Sekhar LN, Stimac D, Bakir A, Rak R. Reconstruction options for complex middle cerebral artery aneurysms. Neurosurgery 2005;56(1 Suppl):66-74

20. Lessne ML, Shah P, Alexander MJ, Barnhart HX, Powers C, Golshani K, et al. Thromboembolic complications after neuroform stent assisted treatment of cerebral aneurysms: the Duke cerebrovascular center experience in 235 patients with 274 stents. Neurosurgery 2011;69:369-375

21. Lopes D, Mangubat E, Keigher K, Cogan C. Intracranial stents in SAH cases. Endovasc Today 2010;August 62-64 